\title{
An experimental study of deep water plunging breakers
}

Marc Perlin and Jianhui He

Department of Naval Architecture and Marine Engineering, University of Michigan, Ann Arbor, Michigan 48109-2145

Luis P. Bernal

Department of Aerospace Engineering, University of Michigan, Ann Arbor, Michigan 48109-2118

(Received 11 January 1996; accepted 2 May 1996)

Plunging breaking waves are generated mechanically on the surface of essentially deep water in a two-dimensional wave tank by superposition of progressive waves with slowly decreasing frequency. The time evolution of the transient wave and the flow properties are measured using several experimental techniques, including nonintrusive surface elevation measurement, particle image velocimetry, and particle tracking velocimetry. The wave generation technique is such that the wave steepness is approximately constant across the amplitude spectrum. Major results include the appearance of a discontinuity in slope at the intersection of the lower surface of the plunging jet and the forward face of the wave that generates parasitic capillary waves; transverse irregularities occur along the upper surface of the falling, plunging jet while the leeward side of the wave remains very smooth and two dimensional; the velocity field is shown to decay rapidly with depth, even in this strongly nonlinear regime, and is similar to that expected from linear theory-the fluid is undisturbed for depths greater than one-half the wavelength; a focusing or convergence of particle velocities are shown to create the jet in the wave crest; vorticity levels determined from the measured, full-field velocity vectors show that the waves are essentially irrotational until incipient breaking occurs; and the magnitude of the largest water particle velocity is about $30 \%$ greater than the phase speed of the (equivalent) linear wave. (c) 1996 American Institute of Physics. [S1070-6631(96)00509-0]

\section{INTRODUCTION}

Breaking waves play a significant role in air-sea interactions such as energy transfer from wind to water, in momentum transfer from waves to currents, and in turbulence generation and turbulence-wave interactions. Knowledge of the hydrodynamics of breaking waves is necessary to improve the design of offshore structures and ships exposed to extreme sea conditions, to understand the dynamics of the upper layer of the oceans, and to facilitate the correct interpretation of remotely sensed data. Breaking, progressive surface waves are usually categorized as spilling, plunging, or combination (spilling-plunging) waves, regardless of the depth of the liquid on which they propagate. Plunging breaking is a dramatic wave phenomenon widely observed on ocean surfaces and is the topic of this experimental effort and discussion. These waves are characterized by the formation of an increasingly protruding and overturning fluid mass at the wave crest, which enters the forward face of the water surface as a jet. Due to their complexity, plunging breaking waves have never been explained adequately and are the focus of the present study.

The breaking process is coupled through wave forces to the design of offshore structures and ships. Also, the dynamics of the upper ocean are clearly influenced and known to be affected by the breaking of surface waves. More recently, the interpretation of remotely sensed data is seen to require knowledge of the breaking process and subsequent shortwave generation. Remote sensing of the oceans and atmosphere by radar, for example, offers the likely possibility of measuring fluid flows on geophysical scales. Since these electromagnetic waves and their backscatter from the ocean surface are believed to be coupled fundamentally with breaking events, it is extremely important to understand the breaking process more fully.

In recent years, theoretical and numerical analyses of near-breaking waves have advanced significantly. LonguetHiggins and Cokelet, ${ }^{1,2}$ using potential theory and a conformal mapping, developed a numerical technique to solve the periodic, two-dimensional, deep water near-breaking-wave problem. Large accelerations in breaking waves were captured using a dynamically controlled time-stepping procedure. A similar method was adopted by Vinje and Brevig ${ }^{3}$ to study the near-breaking-wave problem. They solved numerically for the flow in the physical plane. Tanaka ${ }^{4,5}$ demonstrated that periodic waves with wave steepness greater than approximately 0.43 (the steepness with maximum energy density) are unstable, a discovery that led Jillians ${ }^{6}$ to investigate the evolution of the instability to show how it eventually leads to breaking. Dommermuth and Yue ${ }^{7}$ performed high-resolution computations using a semi-Lagrangian scheme and a regridding algorithm. A numerical, timemarching model to simulate a deep-water breaking wave was used by Skyner et al. ${ }^{8}$ and Skyner and Greated ${ }^{9}$ to compare theory and experiment. Therein, the wave evolution and the velocity field were calculated until the occurrence of jet impingement on the forward face of the wave. Repeated assaults by Peregrine and co-workers (e.g., Dold and Peregrine ${ }^{10}$ ) using computations simulating fully nonlinear, irrotational flows have contributed greatly to our understanding of the near-breaking problem. More recently, Tulin and $\mathrm{Li}^{11}$ outlined a new theoretical analysis of a particular break- 
ing mechanism in which the resonant (sideband) instability is principal. This instability leads to wave breaking within wave groups. This linear stability analysis can be used only to determine the instability band and its initial growth rate. Most recently, Jenkins ${ }^{12}$ provided an analytic solution for a steady (in moving coordinates), plunging breaker that satisfies the surface boundary conditions and uses a Riemann surface so as to avoid the jet reentry problem. In addition, three-dimensional instabilities of steep waves were studied by McLean. ${ }^{13}$ For a complete literature review and discussion of deep water wave breaking through 1993, see the excellent review article by Banner and Peregrine. ${ }^{14}$ Analytically and numerically, however, the deep water (plunging) breaking wave problem and the physics associated with it remain largely unsolved.

Experimental investigation of well-controlled, breakingwave phenomena is essential in the study of breaking waves as theoretical analyses have so-far proved impossible and complete numerical analyses must await faster computers with greatly increased memory. Laboratory experiments using simpler flow configurations than those found in nature can be used to explore characteristics of breaking waves that are much more difficult to capture in field measurements, especially because wave fields are broadbanded in nature. High-quality experimental measurements can be used in direct comparison with theoretical and numerical results. More importantly, measurements can be made in the laboratory to study phenomena that cannot be predicted by mathematical models. Banner and Peregrine ${ }^{14}$ in their review of deep water breaking waves, assert that these experimental studies fall into several distinct categories. Several researchers have concentrated on the detection of breaking and the identification of simple breaking criteria, such as the slope of the wave (Melville; ${ }^{15,16} \mathrm{Koga} ;{ }^{17} \mathrm{Xu} ;{ }^{18}$ Ebuchi et al. $;{ }^{19}$ etc.). However, Melville and Rapp $^{20}$ argue that simple "breaking criteria" based on local wave properties are ambiguous. Their measurements show that the wave slope is reduced as the wave breaks. Other authors such as Kjeldsen and Myrhaug, ${ }^{21}$ and Bonmarin and Ramamonjiarsoa ${ }^{22}$ have investigated the kinematics of breaking waves. A comprehensive study was reported by Bonmarin ${ }^{23}$ on changes in wave steepness and wave speed as the wave approaches breaking. The asymmetry of the wave profile in the near-breaking region, crest evolution, jet formation and overturning, splash-up phenomenon, and modes of air entrainment by the plunging breaker were all investigated. The most systematic work on the deep water breaking wave was reported by Rapp and Melville. ${ }^{24}$ Surface motion, breaking-induced currents, turbulent fluctuations, surface mixing, momentum flux, and energy dissipation were measured in their work.

Although these experimental studies are revealing, fullfield velocity measurements of a plunging breaker are sought herein, as no relationship between the surface displacement and the velocity field is known for strongly nonlinear breaking waves (Melville and $\operatorname{Rapp}^{20}$ ), and only one velocity field measurement of deep water breaking waves has been reported (Skyner and Greated ${ }^{9}$ ). More recently, Skyner $^{25}$, compares numerical predictions and experimental measurements for a plunging breaking wave. (The authors would like to acknowledge one reviewer for pointing out the existence of this work, and thank Dr. Skyner for graciously providing a preprint of his manuscript.) However, as only one experimentally measured velocity field is presented and compared to a numerical simulation (along with a comparison of a time series of the surface evolution), the present effort is complementary and progressive rather than duplicative. In addition, there remains some question in the literature as to the accuracy of the numerical solutions of breaking waves once the forward face of the wave becomes vertical (Schultz et al. ${ }^{26}$ ), and so the discrepancies found by Skyner (even with his diligent effort to produce a wave equivalent to that in the simulation) between numerical and physical experiments are not entirely unexpected, particularly very near breaking. The main goal of this research is to provide a complete set of observations over time and space of the evolution of a deep water plunging, breaking wave. To this end, we report highly resolved measurements of the surface-elevation evolution and the velocity field evolution, from which the vorticity field evolution is calculated. We obtain quantitative and qualitative information for a range of breaking intensities (and breaker types) as determined by a command signal to a mechanical wave generator as explained below; however, we focus only on plunging breakers and present results from a particular example, and note that all of our plungers give similar, qualitative results. Finally, we also investigate the three-dimensionality along the crest of the breaker by using a transverse laser sheet.

A "clean" plunging breaker-that is, without upstream breaking - is required in these experiments, since any previous breaking may contaminate the velocity and vorticity fields in the downstream plunging breaker. Thus, a modification to the Davis and Zarnick ${ }^{27}$ wave generation technique is developed to ensure a clean plunging breaker (see Sec. II for details). A flow visualization technique is used to capture the surface elevation of the breaking wave. Velocities are determined using particle image velocimetry (PIV) and particle tracking velocimetry (PTV). The results of measurements using both techniques are compared with reasonable agreement. The vorticity calculations indicate that the flows are essentially irrotational (outside of the surface boundary layer) up to the jet's reentry into the forward face of the water surface. Using a high-speed imaging system, the local flow field and surface at the crest of the wave are magnified and recorded and short-wavelength growth is observed (i.e., parasitic capillaries) prior to breaking. Transverse perturbations of the wave are seen to occur using a cross-tank laser sheet. The mechanism that generates the transverse roughness across the falling jet is believed to be either stretching of the ambient surface "noise" ubiquitous in any laboratory facility or turbulence-wave interactions, or both. The former phenomenon is the inviscid mechanism described by Longuet-Higgins. ${ }^{28}$

In Sec. II, details are given on modifications made to the Davis and Zarnick technique to generate a clean plunging breaker for the experiments. The laboratory facility, PIV, PTV, and surface measurement techniques are also described in Sec. II. In Sec. III, experimental results are presented and discussed. These include full-field velocity and vorticity 


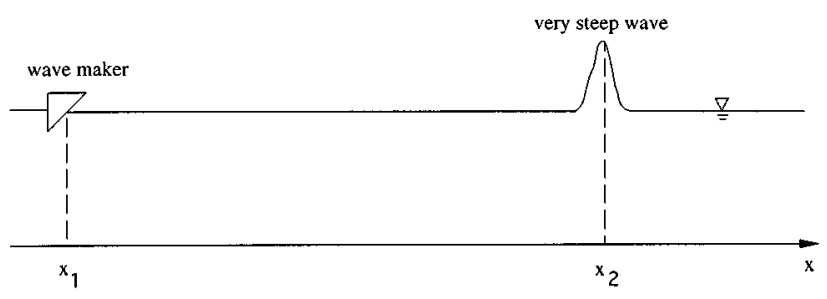

FIG. 1. Schematic of the linear system used to determine the time series at the wavemaker to generate the breaking waves.

measurements as well as the spatial distribution of surface elevations over time. In Sec. IV, conclusions are presented along with a brief discussion of future plans.

\section{EXPERIMENTS}

The experiments are conducted in a two-dimensional wave tank, $35 \mathrm{~m}$ long, $0.7 \mathrm{~m}$ wide, with a water depth of $1.14 \mathrm{~m}$ for the present experiments. Attendant equipment includes a servo-controlled wavemaker with a feedback loop and auxiliary electronics; a high-speed imaging system with a $5 \mathrm{~W}$, argon-ion laser for flow visualization and particle tracking velocimetry; and a twin, Nd-YAG laser system for particle image velocimetry.

The frequency range of waves generated that coalesce to form the breaking wave is 0.8 to $2.0 \mathrm{~Hz}$. Thus, the wave groups are composed of gravity waves. According to linear theory, deep water conditions are realized for these experiments, since the wavelength at the center frequency $(1.4 \mathrm{~Hz})$ is $0.8 \mathrm{~m}$ and the water depth was $1.14 \mathrm{~m}$. (This is borne out by the PIV results, which exhibit no particle velocities at these depths.) A surface skimming system is activated between experiments to remove airborne contaminants from the water surface and a point gauge is used to ensure a constant and repeatable water level.

\section{A. Modifications to the Davis and Zarnick technique for generation of a plunging breaker}

Davis and Zarnick ${ }^{27}$ gave the following mathematical formulation for the generation of a steep wave:

$$
\eta\left(x_{1}, t\right)=\left(\frac{g}{2 \pi\left(x_{2}-x_{1}\right)}\right)^{1 / 2}\left[\cos \left(\sigma^{2}\right) I_{1}+\sin \left(\sigma^{2}\right) I_{2}\right],
$$

where $x_{1}$ is the wave maker location and $x_{2}$ is the breaking wave location, as shown in Fig. 1, and

$$
\begin{aligned}
& I_{1}=\frac{1}{2}-C\left(\sqrt{\frac{2}{\pi}} \sigma\right) ; \\
& I_{2}=\frac{1}{2}-S\left(\sqrt{\frac{2}{\pi}} \sigma\right),
\end{aligned}
$$

with $C(\xi)$ and $S(\xi)$ the usual Fresnel integrals,

$$
C(\xi)=\int_{0}^{\xi} \cos \left(\frac{\pi}{2} y^{2}\right) d y ; \quad S(\xi)=\int_{0}^{\xi} \sin \left(\frac{\pi}{2} y^{2}\right) d y,
$$

and

$$
\sigma=\frac{g^{1 / 2} t}{2\left(x_{2}-x_{1}\right)^{1 / 2}}
$$

These equations give the time history of the water-surface elevation required at the wavemaker to produce a steep wave at position $x_{2}$ in the tank according to linear wave theory. The wavemaker transfer function is required to obtain a command signal. To determine this transfer function, we use a calibrated wave probe placed one wavelength downstream of the wavemaker, oscillate the paddle at distinct frequencies with the same amplitude of the command signal, and measure the generated wave height by recording the output from the probe. The transfer function is calculated throughout the wave frequency range and is used to modify the time series obtained from the Davis and Zarnick technique.

The command-signal time series is further modified by altering the local wave steepness, $k a$, based on a wave period defined by the zero-upcrossing method. That is, each (approximate) local wave period in the group is determined according to the zero-upcrossing method. Then, its radian frequency is calculated and used in the linear dispersion relation to determine the local wave number. Last, its amplitude, $a$, is adjusted so that $k a$ is constant for every zero-upcrossing wave present in the wave group. Therefore, adjusting the gain of the command signal to the wavemaker approximately increases $k a$ uniformly across the entire spectrum. Since $k a$ is usually the small parameter for perturbation expansions for water waves in deep water, we expect that nonlinear effects will be approximately uniform across the spectrum (until the wave field approaches breaking and becomes strongly nonlinear). One advantage of using this technique is that as we change the wavemaker gain, the phase speed of each component is altered approximately equally. To demonstrate that this is so, note that weakly nonlinear wave theory (through third order) gives an amplitude dispersion correction to the frequency of

$$
\omega^{2}=g k\left(1+\frac{(k a)^{2}}{4}\right)
$$

and thus a correction to the nonlinear phase speed of

$$
C_{\text {nonlin }}=C_{\operatorname{lin}}\left(1+\frac{(k a)^{2}}{4}\right)^{1 / 2},
$$

where $C_{\text {nonlin }}$ and $C_{\text {lin }}$ represent the nonlinear and linear phase speeds, respectively. Therefore, the wave components that all have approximately the same value of $k a$ are all affected similarly, and the superposition is approximately intact.

The constant wave steepness throughout the spectrum helps remove premature upstream breaking since the existing nonlinear phase speed effect is (partially) corrected for each wave component. To eliminate premature breaking (spilling), wave components with frequencies higher than $2.0 \mathrm{~Hz}$ or lower than $0.8 \mathrm{~Hz}$ are removed from the time series. The final time series that generates a clean, plunging breaker is presented in Fig. 2. The lowest-frequency amplitude oscillations are limited by wavemaker stroke, as can be seen in Fig. 


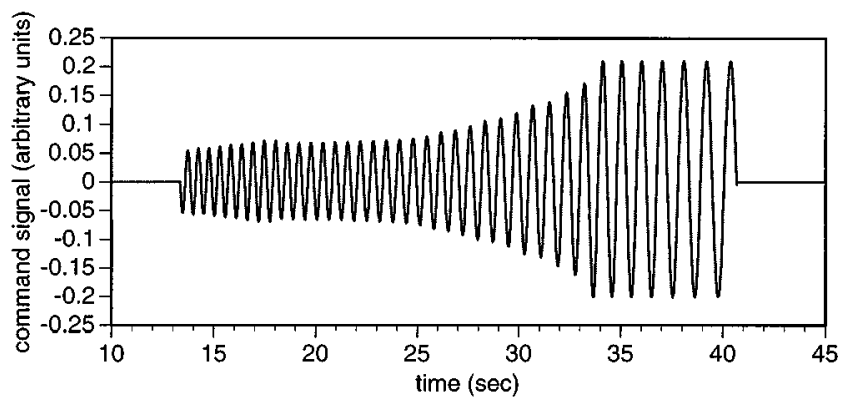

FIG. 2. Command signal input to the wavemaker. Ordinate units are arbitrary.

2 by the uniform-amplitude portion of the time series. Frequency spectra exhibit $k a \approx$ const when measured in close proximity to the wavemaker.

\section{B. Nonintrusive surface-profile measurement technique}

A nonintrusive surface-profile measurement system (Perlin $e t a l .{ }^{29}$ ) is utilized to investigate the surface evolution of deep-water breaking waves. The components of this system include a Spectra-Physics 164, 5W, argon-ion laser as an illumination source; a Kodak Ektapro high-speed video system with an intensified imager capable of framing up to 12 $\mathrm{kHz}$ and gating (shuttering) as fast as $1 \mathrm{MHz}$; and a $50 \mathrm{~mm}$ camera lens. The attendant optics include five dielectric mirrors to redirect the laser beam to the flow field, one spherical lens of focal length $1000 \mathrm{~mm}$, and two cylindrical lenses, with focal lengths 34.0 and $25.4 \mathrm{~mm}$, respectively, to focus and shape the laser beam into a laser sheet with its waist at the quiescent water surface. Recorded images are composed of 239 horizontal pixels by 192 vertical pixels. Fluorescein dye is added to the water as the fluorescing agent for the selected $488.0 \mathrm{~nm}$ wavelength line of the argon-ion laser, which has a power output of approximately $0.7 \mathrm{~W}$ as used.

The plunging breaker is produced with a wedge wavemaker driven by the command signal time series described above. The imager is positioned with its axis oriented in a slightly upward, downstream direction to ensure a good image of the entire flow field. The imager's framing rate is $1000 \mathrm{~Hz}$ and the intensifier's gating rate is $100-300 \mu \mathrm{s}$. To better understand the behavior of the plunging breaker as it steepens toward breaking, we concentrate on the most active region in the wave crest. [This was done in hindsight once the entire flow field had been captured instantaneously using PIV (described below), and it was learned that the flow field was irrotational.] The physical size of the imaged region is approximately $18.5 \mathrm{~cm} \times 12.5 \mathrm{~cm}$. A precision target is used to determine the resolution of the field of view. The horizontal and vertical resolutions are determined to be 0.775 and $0.652 \mathrm{~mm} /$ pixel, respectively.

To increase resolution and capture the parasitic capillary waves, surface elevation measurement is performed at close range by moving the imager closer to the laser sheet. An extension tube of length $25 \mathrm{~mm}$ and a $52 \mathrm{~mm}$ close-up lens are attached to the imager lens. Horizontal and vertical resolutions are determined to be 0.133 and $0.126 \mathrm{~mm} / \mathrm{pixel}$, respectively.

Transverse features are investigated by locating the imager above the water surface and orienting it directly upstream with a cross-tank laser sheet in place. This setup is used to measure the along-crest roughness generated on the plunging jet of liquid. This technique is essentially identical to the measurement of the surface profile, except that the laser sheet is perpendicular to the direction of wave travel, and the location of the sheet is more critical to the success of the measurement. That is, it must penetrate the water surface at the downstream location of the overhanging, plunging jet.

The system timing is achieved by sending a TTL trigger pulse to the high-speed imaging system, at a predetermined "phase" of the breaking wave. The imaging system continuously cycles through its 400 frames of memory until it receives the trigger. A prescribed number of frames (in this experiment, 200) are recorded after the trigger is received, with the previous 200 frames already resident in memory. The 400 images are downloaded to a computer via a standard GPIB interface and are ready for analysis.

\section{Particle image velocimetry}

The velocity field in the breaking waves is measured using particle image velocimetry (PIV). This system is a modified version of the PIV system used by Aissi and Bernal $^{30}$ and more recently by Shack, Bernal, and Shih. ${ }^{31}$ The flow is illuminated with twin Nd:YAG lasers each delivering more than $320 \mathrm{~mJ}$ at $1064 \mathrm{~nm}$ in a $6 \mathrm{~ns}$ pulse. The output beam from the lasers are combined in a single coaxial beam and frequency doubled to $532 \mathrm{~nm}$. The resulting green beams are shaped into a plane sheet of light by a $1000 \mathrm{~mm}$ focal length spherical lens and a $25.4 \mathrm{~mm}$ focal length cylindrical lens. The thickness of the light sheet at the measurement location is less than $0.5 \mathrm{~mm}$ and the overlap of the light sheets formed by each laser is adjusted to within a fraction of the laser sheet thickness. The sheet forming optics are located above the wave tank, as shown schematically in Fig. 3. Thus, the laser light sheet enters the water from above, resulting in significant refraction effects at the water surface for large surface deformation. The sheet is oriented along the centerplane of the wave tank at the location of breaking. The axial extent of the illuminated region is $0.7 \mathrm{~m}$. Two images of the flow are recorded on the same photograph by firing the two Nd-YAG lasers in close sequence. A delay generator is used to trigger the second laser from the input trigger signal. The actual time between exposures is measured for each image, with a resolution better than $1 \mu \mathrm{s}$. For the present measurements, the time between exposures is $2.57 \mathrm{~ms}$.

The flow is seeded with titanium dioxide $\left(\mathrm{TiO}_{2}\right)$ particles of $2.8 \mu \mathrm{m}$ mean diameter. The estimated terminal velocity (i.e., Stokes settling velocity) of the particles is $0.012 \mathrm{~mm} / \mathrm{s}$. Terminal velocities of this magnitude resulted in significant settling of the particles. To minimize this effect, the particles are stirred to more uniformly distribute them, and the water is allowed to settle for five minutes prior to each run. There is an additional 10 min wait after a breaking event, but prior to stirring that is required to allow the motion induced by the 


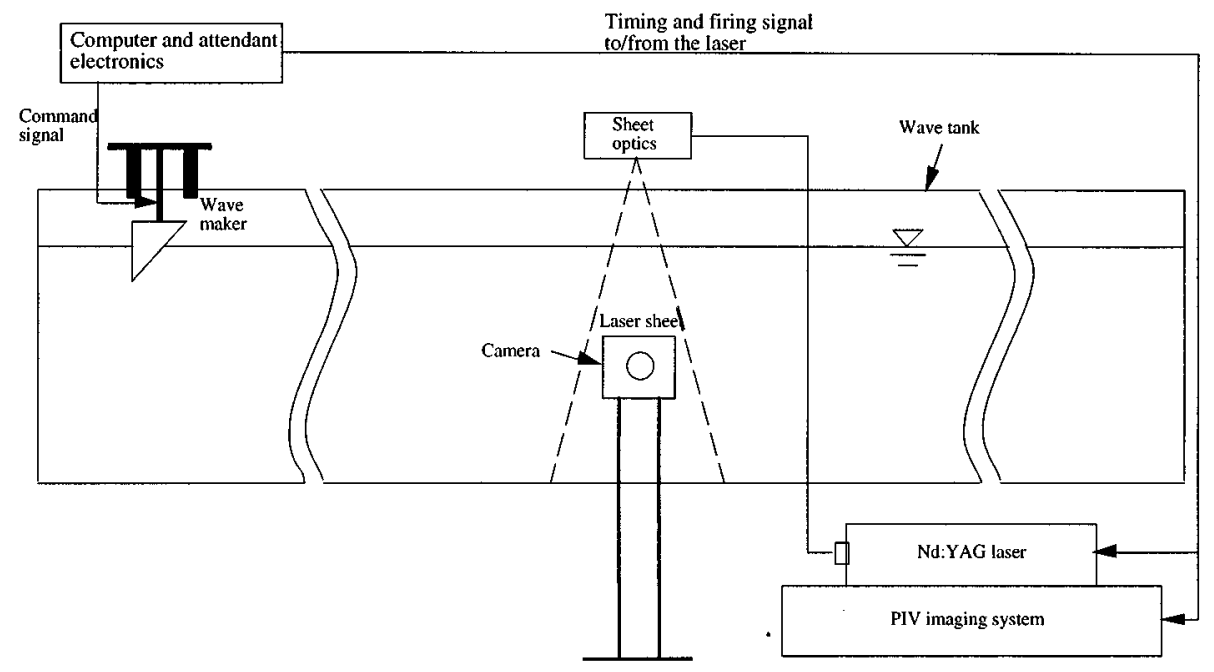

FIG. 3. Schematic of the PIV measurement system used.

breaking event and nonbreaking wave motions to subside. To ensure that all motions induced by the stirring and by the wave generation/breaking process have decayed to quiescent levels during this temporal window (15 min in total), images are recorded and particle velocities/vorticities are determined, as is the surface elevation. A comparison to these same quantities obtained after the facility is left undisturbed for twice this period and four times this period showed quantitatively that the disturbances had indeed subsided to quiescent levels. (In these latter tests, initially, in addition to the particles that remained in the water column, particles are placed on the surface from immediately above it and allowed to settle naturally.) The particle images are recorded on Kodak T-Max 400 film using a 4 in. $\times 5$ in. photographic camera. The camera is equipped with a $135 \mathrm{~mm}$ focal length lens and the pictures are obtained at an aperture $f \# 4.7$. The distance between the camera lens and the laser sheet is set at approximately $1.85 \mathrm{~m}$ and the optical magnification is 0.079 . A scanning mirror is used to introduce a displacement bias (image shift) on the particle images (Adrian ${ }^{32}$ ). The angular velocity of the scanning mirror is adjusted to produce a bias displacement that corresponds to a flow velocity of $1 \mathrm{~m} / \mathrm{s}$ in the direction of the wave motion. The magnification and the bias displacement are measured directly on each image using a reference scale located in the field of view above the water surface.

A critical aspect of the velocity measurements is synchronization between the wavemaker and the PIV imaging system. This is to ensure that the images are recorded at the proper phase of the wave breaking process. To accomplish this in the present experiments, the computer used to generate the command signal to the wavemaker is also used to generate simultaneously a TTL master clock pulse to control the PIV system. This computer generated master clock signal is used to drive the scanning mirror, which in turn controls the Nd-YAG lasers' firing sequence. The delay between the wavemaker motion and the triggering of the lasers is calculated on $10 \mathrm{~ms}$ intervals with a resolution better than $1 \mathrm{~ms}$ in the range 56.85-57.40 s used in these experiments. The sys- tem synchronization is monitored by an oscilloscope.

The PIV images are processed using the Young's fringes interrogation technique. The system used is described by Bernal and Kwon ${ }^{33}$ and Aissi and Bernal. ${ }^{30}$ For the present measurements, the flow field is interrogated in a rectangular region $240 \times 750 \mathrm{~mm}$. Low and high resolution scans are conducted with resolution of 15 and $7.5 \mathrm{~mm}$, respectively. The corresponding datasets contained measurements on an equally spaced grid of $51 \times 11$ points and $101 \times 13$ points, respectively. Thus, vector fields with 1874 velocity vectors are obtained for each image. Although an automatic analysis routine is available, in the present measurement manual processing is used to improve the quality of the results. The uncertainty of the velocity measurement is approximately $2.0 \mathrm{~cm} / \mathrm{s}$. This value is determined from PIV images of the quiescent tank using the same data acquisition procedures.
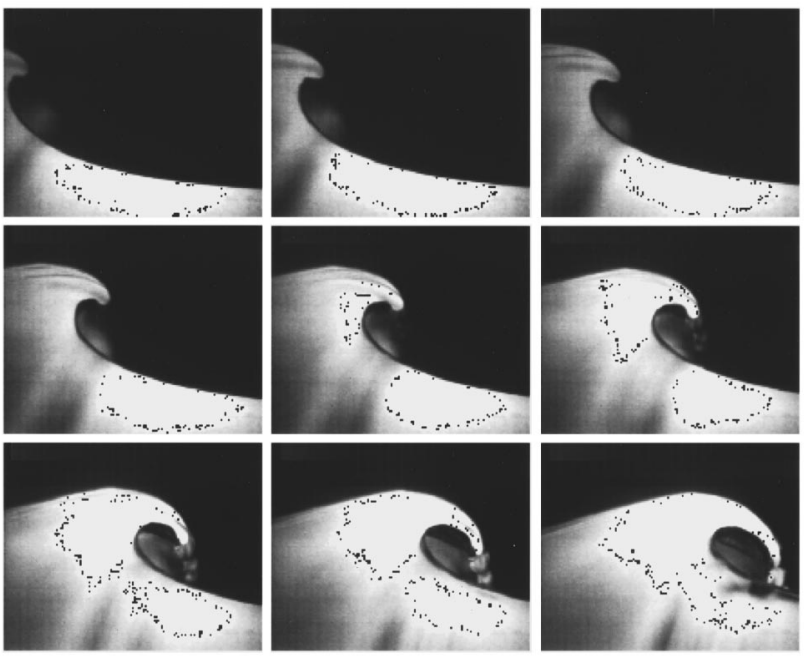

FIG. 4. Time series of the plunging breaker showing the formation, overturning, and elongation of the jet. The vertical and horizontal resolutions are 0.652 and $0.775 \mathrm{~mm} /$ pixel, respectively, with a time interval between frames shown of $0.01 \mathrm{~s}$. Each image is $155.8 \mathrm{~mm}$ horizontal by $148.8 \mathrm{~mm}$ vertical. 

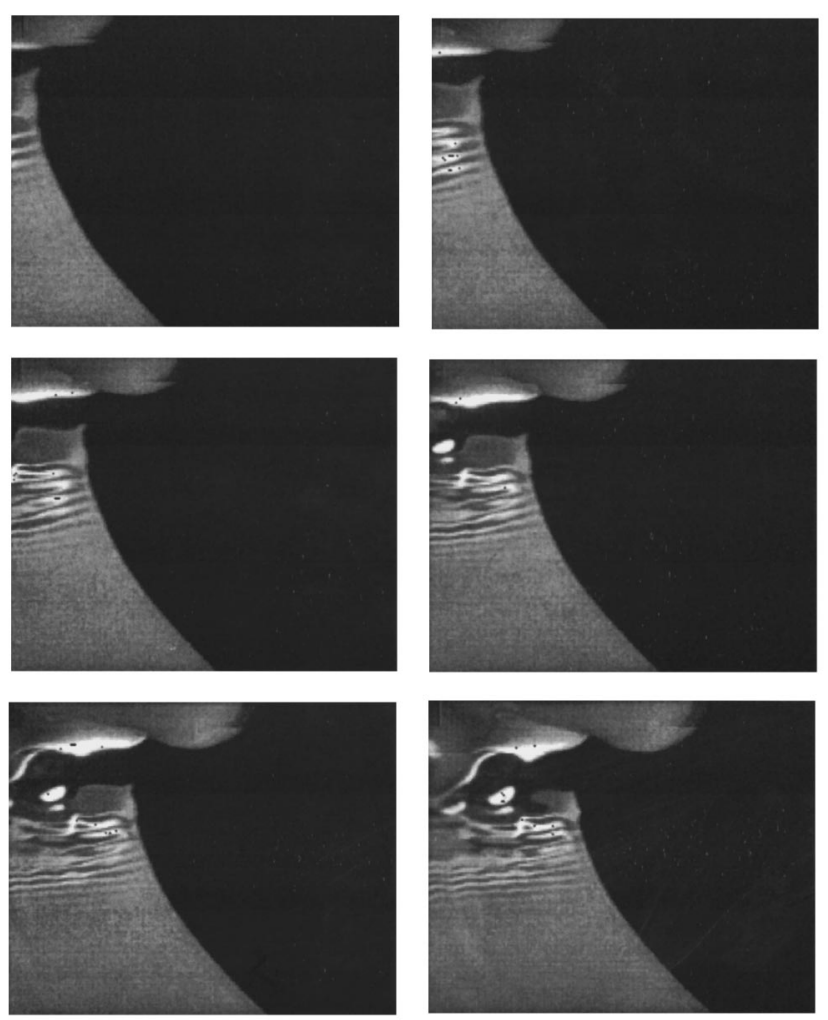

FIG. 5. Parasitic capillary waves observed on the lower front face of the plunger. The vertical and horizontal resolutions are 0.126 and $0.133 \mathrm{~mm} /$ pixel, respectively, with a time interval between frames shown of $0.002 \mathrm{~s}$. Each image is $30.11 \mathrm{~mm}$ horizontal by $25.54 \mathrm{~mm}$ vertical.

\section{Particle tracking velocimetry}

The velocity at the wave crest is measured using particle tracking velocimetry (PTV). In this case, the velocity is determined from the length of particle traces recorded in long time exposures of the flow. The water is seeded with silvercoated hollow glass spheres of $10 \mu \mathrm{m}$ mean diameter and 1.3 $\mathrm{g} / \mathrm{cm}^{3}$ density. The concentration of the particles is adjusted to provide sufficient data without making identification of individual particle traces difficult.

The PTV images are obtained using the Kodak Ektapro high-speed video system and the Spectra-Physics 164 argonion laser described above. A $5 \mathrm{~cm} \times 4 \mathrm{~cm}$ region of the flow is imaged using a $100 \mathrm{~mm}$ lens attached to a $15 \mathrm{~mm}$ extension tube. The horizontal and vertical resolutions are 0.224 and $0.229 \mathrm{~mm} / \mathrm{pixel}$, respectively. To ensure the longest possible streaks, the exposure time used is $5 \mathrm{~ms}$ and the framing rate is $125 \mathrm{~Hz}$. The intensifier gain and the camera lens aperture are adjusted to properly expose the video image of the silver-coated particles. The system timing used in the surface profile measurements is also used in the PTV measurements. The resolution of the velocity magnitude as measured by PTV is estimated to be $4.5 \mathrm{~cm} / \mathrm{s}$.

\section{RESULTS}

\section{A. Flow visualization results}

As our objective is to provide a set of experiments of a deep water plunging, breaking wave, we measure highly re-
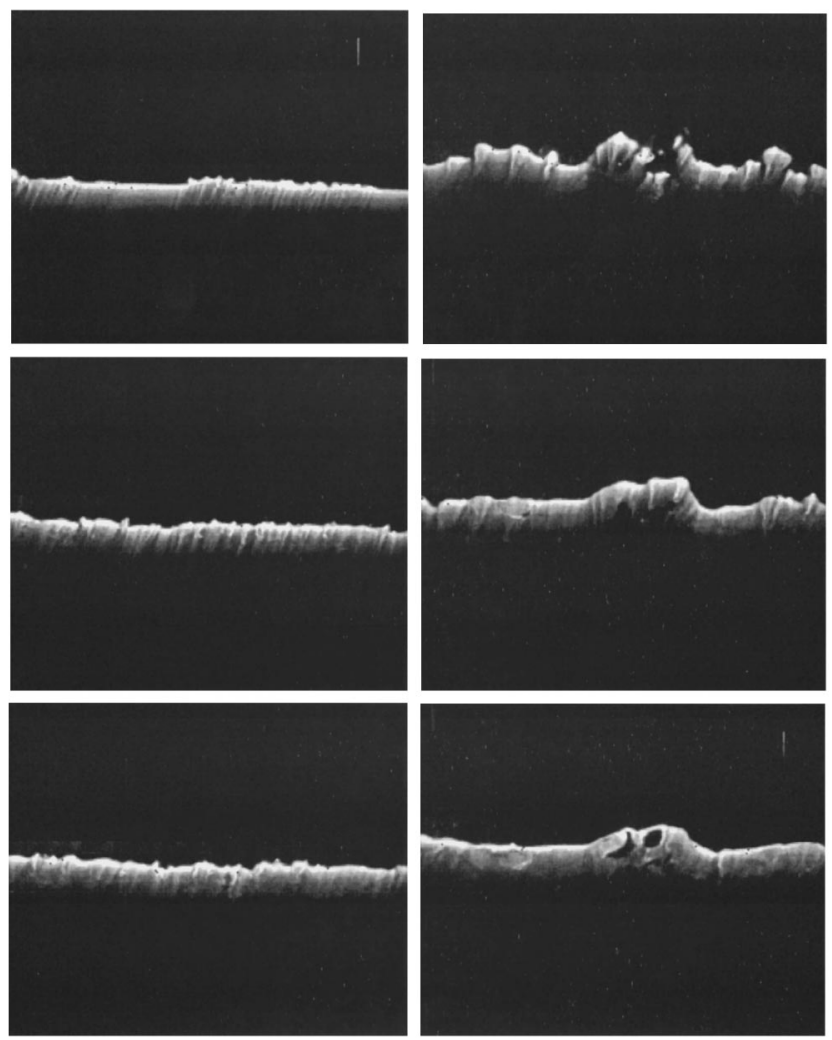

FIG. 6. Transverse irregularities for a time series with a $2 \mathrm{~ms}$ interval. The vertical and horizontal scales of these images are approximately 8 and 10 $\mathrm{cm}$ respectively, and the images shown are the falling jet's upper surface only-the leeward side of the wave crest is always smoothly varying (although not shown).

solved surface elevations and velocity fields, from which the vorticity field is calculated. Although we obtain quantitative and qualitative information for several breaking intensities (and all breaker types), as determined by the voltage level of our command signal to the wave generator, we focus only on plunging breakers and present results from a particular example, as all plungers give qualitatively similar results.

A time series of the plunging breaking waves is shown in Fig. 4 in which the time interval between images presented is $0.01 \mathrm{~s}$. In the first stage of plunging, the wave front becomes vertical. A two-dimensional jet with a rounded end is then formed at the uppermost part of the vertical wave front. Note that a kink or discontinuity in slope is formed where the underside of the jet intersects the front face of the wave. The jet outpaces the wave, overturning as it progresses. It lengthens as it is "fed" from the primary flow, and it approaches the forward face of the wave. Prior to the jet impingement on the forward face, the discontinuity in slope vanishes. That is, as is made evident by the laser illumination at least, a smooth, two-dimensional jet is incident on the forward surface. The jet impingement on the water surface is followed by a splash-up process (not shown). The entire process, from jet formation to reentry, occurs within $0.1 \mathrm{~s}$. The wave "period" is on the order of $0.7 \mathrm{~s}$. Thus, plunging breaking is a local phenomenon in that it does not involve the entire wave. This process is generally in accordance with the results of Bonmarin. ${ }^{23}$ 


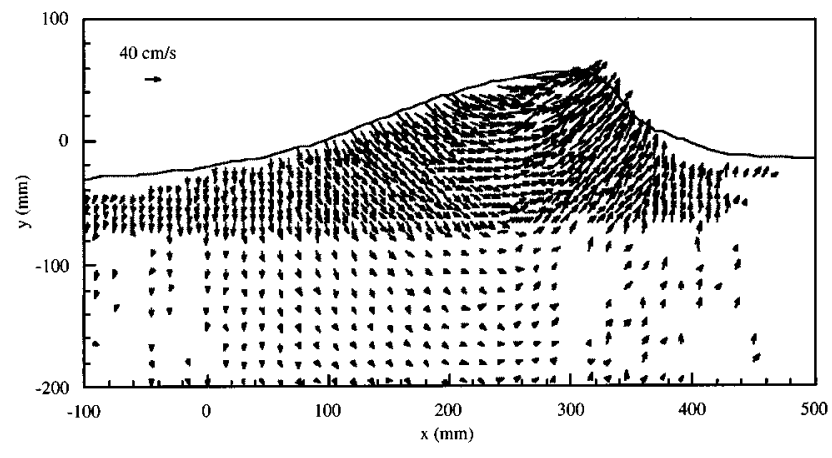

FIG. 7. Velocity field at $t=57.20 \mathrm{~s}$, prior to overturning.

Parasitic capillary waves are observed to occur along the highest elevations of the lower front face of the plunging wave in our magnified view (see Fig. 5). The overhanging plunger is visible in the uppermost portion of these images, too. These images are presented at intervals of $0.002 \mathrm{~s}$ and therefore the entire set of images spans about the interval between each pair of images in Fig. 4. The parasitic capillaries are generated when the front face becomes approximately vertical and the jet develops. These small waves can be seen in the first and second images of Fig. 4, also, and are located just beneath the discontinuity in slope discussed above. Initially, as the projecting jet is formed at the wave crest, increasing numbers of capillary waves (with decreasing wavelength and amplitude as a function of distance from the kink) are observed on the front face; however, as the jet lengthens and falls toward the forward face, the kink disappears, as do the parasitic capillaries, leaving a smoothly varying surface connecting the jet's underside and the front face of the wave.

In Fig. 6, we present a series of images that exhibit transverse perturbations. These images are recorded using the transverse laser sheet setup and are shown at intervals of $0.002 \mathrm{~s}$. The irregularities occur on the upper surface of the jet as it falls toward the forward surface. They do not occur on the leeward side of the crest, as is seen in later images (not presented). These transverse irregularities could be the disintegration of the jet, as explained in Longuet-Higgins, ${ }^{28}$ they could be the manifestation of turbulence-wave interactions, or they could be a combination of both. Small, random ambient disturbances always present on the water surface may be the source of the transverse waves required by the Longuet-Higgins' theory. Many realizations of the transverse laser sheet are conducted; however, the results are not repeatable, as expected for either mechanism, since they are due to random, small surface irregularities present prior to the arrival of the plunging breaker or due to turbulence. Thus, the nonrepeatability supports both suppositions; however, interestingly, these irregularities are never recorded in any longitudinal images (such as those in Fig. 4) and so it is less likely that they are induced by turbulence.

\section{B. PIV and PTV results}

The wavelength of the plunging breaker is approximately $0.7 \mathrm{~m}$, based on the "zero-downcross analysis" used

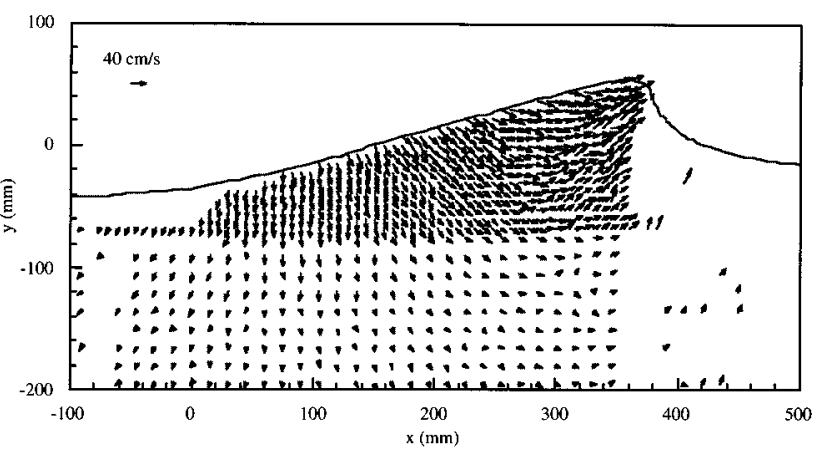

FIG. 8. Velocity field at $t=57.25 \mathrm{~s}$, prior to overturning.

by Bonmarin. ${ }^{23}$ To provide a basis for comparison during analysis, we calculate the phase speed of the wave at this wavelength using linearized wave theory. The resulting wave phase speed is $1.05 \mathrm{~m} / \mathrm{s}$. An approximate, measured phase speed of the plunging breaker is computed by determining the distance between two wave surfaces formed by the double exposures on a PIV photograph. At $1.08 \mathrm{~m} / \mathrm{s}$, the measured phase speed is surprisingly close to its linear theory approximation, albeit fortuitous.

A time series of three velocity vector plots for the plunging breaker are shown in Figs. 7, 8, and 9. The corresponding photographs are taken at times of $57.20 \mathrm{~s}, 57.25, \mathrm{~s}$ and 57.35 $\mathrm{s}$, respectively, after the wavemaker motion is initiated. The first two figures present pre-breaking velocity vector plots, the third figure presents a post-breaking case. A magnified (local) velocity field of the wave crest that corresponds to Fig. 7 is shown in Fig. 10. The maximum laser sheet width we can achieve at the free surface is limited to about $0.7 \mathrm{~m}$, as a wider sheet does not supply sufficient laser light intensity to expose the film. Thus, the vector plots are $0.7 \mathrm{~m}$ or less. As the wave evolves and becomes steep and breaks, the particle images as captured in this study deteriorate due to the violent surface deformations. Our full-field measurements suffer degradation, especially downstream, as the wave breaks, as well during the post-breaking phase. This is clearly seen by the velocity vectors presented in Figs. 8 and 9.

As can be seen in Figs. 7, 8, and 9, the velocity magnitude decays with water depth, as expected. For depths greater

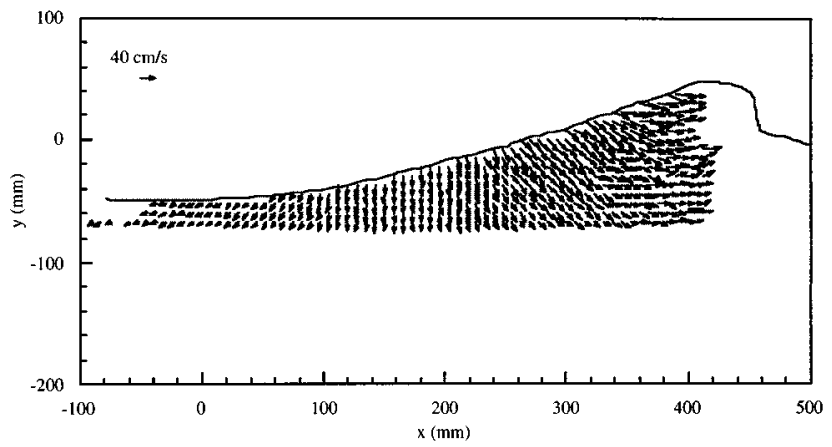

FIG. 9. Velocity field at $t=57.35 \mathrm{~s}$, with the front face nearly vertical. 


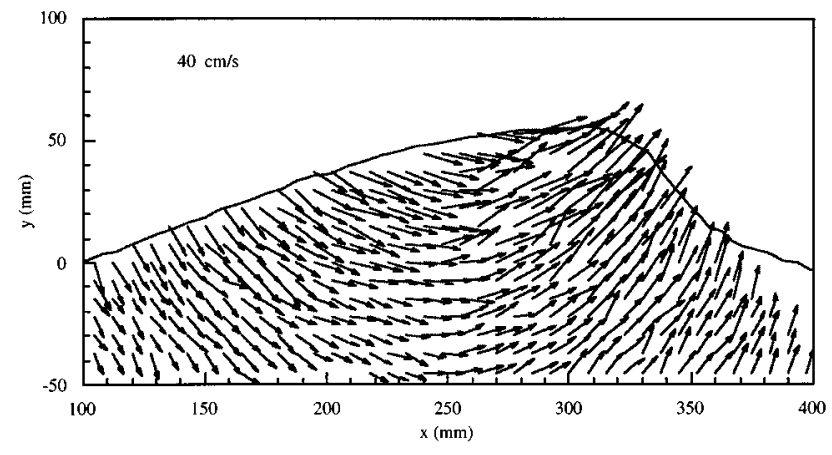

FIG. 10. Magnified view of the velocity field at $t=57.20 \mathrm{~s}$.

than $30 \mathrm{~cm}$ with respect to the mean water level, the magnitudes of the velocity vectors are essentially zero. Thus, plunging breaking has an insignificant influence on particle motions at depths greater than half the wavelength (as calculated from linear theory using an estimate of the wave period and length). In this respect, a plunging breaking wave demonstrates similar behavior to a deep water, plane progressive wave.

A close inspection of Fig. 7 reveals that the wave develops a convergence-like motion toward the steepening wave front as the wave approaches breaking. The largest velocities are located at the wave crest and the uppermost part of the wave front, and their magnitudes are of the order of $1 \mathrm{~m} / \mathrm{s}$. However, the largest horizontal particle speed, a critical parameter in the phenomenon of jet formation and overturning, captured in our PIV images is about $0.8 \mathrm{~m} / \mathrm{s}$. It is usually acknowledged that water particles develop velocity magnitudes greater than the wave phase speed when an ejecting jet is formed at the wave front. Indeed, we show momentarily in our PTV images that the largest (horizontal) particle speeds are greater than the phase speed. Our full-field measurements either did not have sufficient resolution very close to the air-water interface, or due to settling of the particles near the surface, no particles were in the proper location, to capture the largest particle speeds, which are situated at the emerging jet.

The measured velocity fields are used to determine the vorticity fields of the flow. The vorticity is evaluated using the well-known equation of circulation, which yields

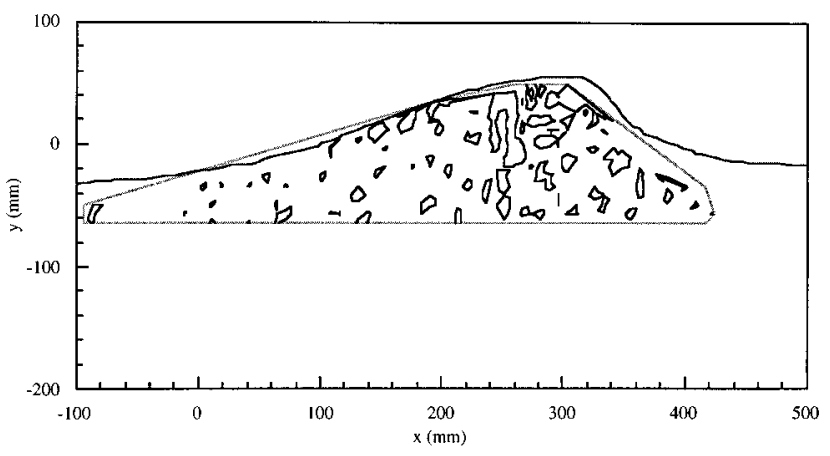

FIG. 11. Graph of the vorticity contours at $t=57.20 \mathrm{~s}$. The contour increment is $26 \mathrm{rad} / \mathrm{s}$.

$$
\omega=\frac{1}{A} \int \vec{u} \cdot \vec{d} l,
$$

where the circulation integral is evaluated at each point using data from eight neighboring points. The ambient flow in the quiescent wave tank is used to determine the level of vorticity that constitutes background noise. A maximum vorticity level of approximately $20 \mathrm{rad} / \mathrm{s}$ is found. A typical vorticity contour plot $(t=57.20 \mathrm{~s})$ is shown in Fig. 11. The prebreaking vorticity is of the same order as that of the quiescent facility. (There is a maximum of about twice the ambient vorticity in the pre-breaking velocity field.) The vorticity along the leeside of the wave is within the ambient noise level also, and is thus insignificant, even immediately after breaking. The flow is essentially irrotational to breaking. Thus, potential theory is valid (outside the surface boundary layer) and may be used until actual breaking occurs in a plunging breaker.

Figure 12 presents two typical PTV images that are recorded with a gating rate of $200 \mathrm{~Hz}$ and a framing rate of 125 $\mathrm{Hz}$. The particle velocity is calculated by measuring the particle streak length and dividing it by the exposure time. These velocity vectors are compared with the PIV results for the same phase at comparable locations in the flow field, especially in the vicinity of the wave crest. The PTV results obtained agree with our PTV measurements, as well as provide interesting data that are unavailable from the PIV images. The particle velocities become almost horizontal and their magnitudes are on the order of the phase speed as the

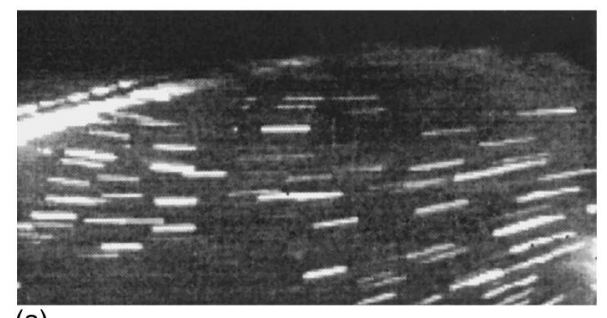

(a)

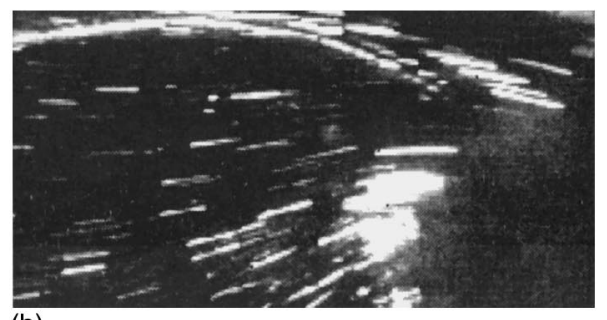

(b)

FIG. 12. Images of particle streaks with horizontal and vertical resolutions of 0.233 and $0.233 \mathrm{~mm} / \mathrm{pixel}$ in (a) and 0.215 and $0.225 \mathrm{~mm} / \mathrm{pixel}$ in (b), respectively. Image (b) is recorded farther downstream. A gating rate of $200 \mathrm{~Hz}$ and a framing rate of $125 \mathrm{~Hz}$ are used. The image shown in (a) is $55.69 \mathrm{~mm}$ horizontal by $44.74 \mathrm{~mm}$ vertical. The image shown in (b) is $51.39 \mathrm{~mm}$ horizontal by $43.20 \mathrm{~mm}$ vertical. 
wave front nears vertical. At this juncture, the water particles at the crest begin to accelerate as they receive momentum from the water particles behind and beneath them. An ejecting jet is then formed at the crest with its particles moving forward faster than the wave itself. The largest particle speed captured is situated at the vertex of the overturning jet, with a horizontal velocity $30 \%$ greater than the phase speed. Also, note in Fig. 12(b), that there exists a region of bright intensity where the particle streaks are combined and indistinguishable; this region corresponds to the location of the convergence-like motion discussed previously and visible in Figs. 7 and 10.

\section{CONCLUDING REMARKS}

The superposition technique of Davis and Zarnick is modified to generate a "clean" plunging breaking wave. A transfer function is applied such that the wave steepness, $k a$, for every frequency present, is constant. As signal gain is altered, the wave steepness is affected uniformly across the spectrum. This removed any premature upstream breaking since the existing nonlinear effect is approximately the same for each wave component. As expected, it is found that as the gain to the wavemaker is increased (i.e., only the magnitude of the command signal is altered), the breaking wave is changed dramatically. The large wave at the superposition location is initially steep, but nonbreaking. Increasing the command signal slightly causes a spilling breaker to occur. A further increase in signal strength generates a spillingplunging breaking wave. Another increase in signal gain generates a plunging breaker. In this manner, complete control over the breaker type is exercised and repeatable wave forms are possible. This plunger is investigated by flow visualization, particle image velocimetry (PIV), and particle tracking velocimetry (PTV). Full-field velocity measurements are obtained and the vorticity fields are calculated.

Plunging breaking is captured by flow visualization using a high-speed imaging system. An asymmetric wave profile is captured during pre-breaking. A small jet is seen forming at the peak of the wave front, accelerating forward more quickly than the wave itself, overturning, becoming incident on the water surface, and finally creating a splash zone. A magnified image captures the appearance of parasitic capillary waves on the upper part of the wave front as the wave front becomes vertical. Additional capillary waves are formed as the wave propagates and the kink between the lower jet surface and the wave front remains. The discontinuity in slope as well as the parasitic capillaries disappear as the jet and wave continue to evolve.

Transverse irregularities are observed along the forward portion of the plunging jet. However, the flow along the leeward side of the wave remains two dimensional during the initial stages of breaking. Interestingly, longitudinal surface profiles acquired using a laser sheet exhibit a form that appears quite two dimensional, leading to the conclusion that the perturbations are somewhat regular in the longitudinal direction.

Full-field velocity measurement is achieved by particle image velocimetry. The measured velocity field at a depth of $30 \mathrm{~cm}$ is essentially undisturbed by the wave's presence.
That is, the plunging breaker has an insignificant effect on water motion at a depth greater than half its wavelength. Velocity vectors converge in the vicinity of the steepening wave front. The vorticity is calculated based on the measured velocity field. The flow is essentially irrotational to the break point; thus, potential theory is valid (outside the surface boundary layer) until breaking ensues.

A comparison of the Skyner ${ }^{25}$ PIV-measured particle velocities (his Fig. 11) with our measurements reveals a noticeable difference. (Noting that the two signals to the wavemakers are markedly different and that the particular phase of the breaking wave may be different, there is no reason to expect that the energetics of the two waves are similar, although both are plunging breakers. Nonetheless, we may compare qualitatively.) Specifically, our results show that from the rear side of the crest to the forward face of the crest, the instantaneous particle velocities are in a circular-like motion, whereas the Skyner velocities are nearly horizontally oriented near the rear portion of the crest and are more similar to ours near the front face of the crest. Direct comparison of breaking wave experiments is extremely difficult, and will remain so until a "breaker parameter" is determined for deep water waves that addresses the coalescence of energy, for example.

The measurements obtained by PTV are in line with the results of the PIV measurements. The largest velocity magnitude is located in the overturning jet and its horizontal component is $30 \%$ greater than the phase speed of the wave.

In summary, we have measured the evolution of a plunging breaking wave generated by superposition of progressive waves of increasing wavelength and constant steepness. Capillary waves form on the forward face of the wave and have been documented. The main feature of the flow is the formation of the overhanging jet, which is shown here to result from convergence of the water motion near the top of the wave crest. The motion is essentially irrotational to the break point. The water surface of the falling jet is shown to have large transverse irregularities of short wavelength; however, aside from the capillaries and slope discontinuity that appear shortly after its formation, its profile is well behaved and smooth in the direction of wave motion. The present study has focused on the surface profile to the point of jet impingement and on the flow field to the point of jet formation. Important issues that remain to be addressed in laboratory experiments are the flow within the jet and the flow in the post-breaking turbulent region. These aspects of the flow are particularly challenging to characterize experimentally. However, they are the key to further validate increasingly sophisticated and detailed theoretical and numerical studies of plunging breaking waves.

\section{ACKNOWLEDGMENTS}

The support of the Office of Naval Research under Contract No. N00014-92-J-1750, Dr. Edwin P. Rood program monitor, is appreciated greatly.

\footnotetext{
${ }^{1}$ M. S. Longuet-Higgins and E. D. Cokelet, "The deformation of steep surface waves on water. I. A numerical method of computation,' Proc. R. Soc. London Ser. A 358, 1 (1976).
} 
${ }^{2}$ M. S. Longuet-Higgins and E. D. Cokelet, "The deformation of steep surface waves on water. II. Growth of normal mode instabilities,' Proc. R. Soc. London Ser. A 364, 1 (1978).

${ }^{3}$ T. Vinje and P. Brevig, "Numerical simulation of breaking waves,' Adv. Water Res. 4, 77 (1981).

${ }^{4}$ M. Tanaka, 'The stability of steep gravity waves,' J. Phys. Soc. Jpn. 52, 3047 (1983).

${ }^{5}$ M. Tanaka, "'The stability of steep gravity waves. II,' J. Fluid Mech. 156, 281 (1985).

${ }^{6}$ W. J. Jillians, “The superharmonic instability of Stokes waves in deep water,' J. Fluid Mech. 204, 563 (1989).

${ }^{7}$ D. G. Dommermuth, D. K.-P. Yue, W. M. Lin, R. J. Rapp, E. S. Chan, and W. K. Melville, “Deep-water plunging breakers: A comparison between potential theory and experiments,' J. Fluid Mech. 189, 423 (1988).

${ }^{8}$ D. J. Skyner, C. Gray, and C. A. Greated, “A comparison of time-stepping numerical predictions with whole-field flow measurement in breaking waves,' Water Wave Kinematics, edited by A. Torum and O. T. Gudmestad, 1990, p. 491.

${ }^{9}$ D. J. Skyner and C. A. Greated, "The evolution of a long-crested deepwater breaking wave,', 2nd Int. Off. Polar Eng. Conf. 111, 132 (1992).

${ }^{10}$ J. W. Dold and D. H. Peregrine, "Water-wave modulation," Proc. 20th Int. Conf. Coastal Eng. 1, 163 (1986).

${ }^{11}$ M. P. Tulin and J. J. Li, ' On the breaking of energetic waves,' Int. J. Off. Polar Eng. 2, 46 (1992).

${ }^{12}$ A. D. Jenkins, "A stationary potential-flow approximation for a breakingwave crest,', J. Fluid Mech. 280, 335 (1994).

${ }^{13}$ J. W. McLean, "Instabilities of finite-amplitude water waves,' J. Fluid Mech. 114, 315 (1982).

${ }^{14}$ M. L. Banner and D. H. Peregrine, "Wave breaking in deep water," Annu. Rev. Fluid Mech. 25, 373 (1993).

${ }^{15}$ W. K. Melville, "The instability and breaking of deep-water waves,' J. Fluid Mech. 115, 165 (1982).

${ }^{16}$ W. K. Melville, "Wave modulation and breakdown,' J. Fluid Mech. 128, 489 (1983).

${ }^{17}$ M. Koga, "Characteristics of a breaking wind-wave field in the light of the individual wind-wave concept,', J. Ocean Soc. Jpn. 40, 105 (1984).

${ }^{18}$ D. Xu, P. A. Hwang, and J. Wu, 'Breaking of wind-generated waves,' J. Phys. Ocean. 16, 2172 (1986).
${ }^{19}$ N. Ebuchi, H. Kawamura, and Y. Toba, "Fine structure of laboratory wind-wave surfaces studied using an optical method," Boundary Layer Meteorol. 39, 133 (1987).

${ }^{20}$ W. K. Melville and R. J. Rapp, "The surface velocity field in steep and breaking waves,' J. Fluid Mech. 189, 1 (1988).

${ }^{21}$ S. P. Kjeldsen and D. Myrhaug, "Kinematics and dynamics of breaking waves,' Ships in Rough Seas, Part 4 (1978).

${ }^{22}$ P. Bonmarin and A. Ramamonjiarsoa, "Deformation to breaking of deep water gravity waves,' Exp. Fluids 3, 11 (1985).

${ }^{23} \mathrm{P}$. Bonmarin, "Geometric properties of deep-water breaking waves," J. Fluid Mech. 209, 405 (1989).

${ }^{24}$ R. J. Rapp and W. K. Melville, "'Laboratory measurements of deep-water breaking waves,' Philos. Trans. R. Soc. London Ser. A 331, 735 (1990).

${ }^{25}$ D. Skyner, "A comparison of numerical predictions and experimental measurements of the internal kinematics of a deep-water plunging wave," J. Fluid Mech. (in press).

${ }^{26}$ W. W. Schultz, J. Huh, and O. M. Griffin, "Potential energy in steep and breaking waves,'” J. Fluid Mech. 278, 201 (1994).

${ }^{27}$ M. C. Davis and E. E. Zarnick, "Testing ship models in transient waves,", Fifth Symposium on Naval Hydrodynamics, 1964, p. 507.

${ }^{28}$ M. S. Longuet-Higgins, "On the disintegration of the jet in a plunging breaker,', J. Phys. Ocean. 25, 2458 (1995).

${ }^{29}$ M. Perlin, H. J. Lin, and C. L. Ting, “On parasitic capillary waves generated by steep gravity waves: An experimental investigation with spatial and temporal measurements,', J. Fluid Mech. 255, 597 (1993).

${ }^{30} \mathrm{~S}$. Aissi and L. P. Bernal, "PIV investigation of an aperiodic forced mixing layer,' AIAA Paper No. 93-3241, 1993.

${ }^{31}$ D. H. Shack, L. P. Bernal, and G. S. Shih, "Experimental investigation of underhood flow in a simplified automobile geometry,' Proceedings of the ASME Fluids Engineering Division Summer Conference, 1994.

${ }^{32}$ R. J. Adrian, "Image shifting technique to resolve directional ambiguity in double-pulsed velocimetry,', Appl. Opt. 25, 3855 (1986).

${ }^{33}$ L. P. Bernal and J. T. Kwon, "Surface flow velocity field measurement by laser speckle photography," Proceedings of the 4th Intern Symposium on Application of Laser Anemometry to Fluid Mechanics, Lisbon, 1988. 